\title{
EMBEDDINGS OF COMPLEX LINE SYSTEMS AND FINITE REFLECTION GROUPS
}

\author{
MURALEEDARAN KRISHNASAMY and D. E. TAYLOR ${ }^{\bowtie}$
}

(Received 19 December 2007; accepted 19 April 2008)

Communicated by Martin W. Liebeck

Dedicated to Cheryl Praeger for her sixtieth birthday

\begin{abstract}
A star is a planar set of three lines through a common point in which the angle between each pair is $60^{\circ}$. A set of lines through a point in which the angle between each pair of lines is 60 or $90^{\circ}$ is star-closed if for every pair of its lines at $60^{\circ}$ the set contains the third line of the star. In 1976 Cameron, Goethals, Seidel and Shult showed that the indecomposable star-closed sets in Euclidean space are the root systems of types $A_{n}, D_{n}, E_{6}, E_{7}$ and $E_{8}$. This result was a key part of their determination of all graphs with least eigenvalue -2 . Subsequently, Cvetković, Rowlinson and Simić determined all star-closed extensions of these line systems. We generalize this result on extensions of line systems to complex $n$-space equipped with a hermitian inner product. There is one further infinite family, and two exceptional types arising from Burkhardt and Mitchell's complex reflection groups in dimensions five and six. The proof is a geometric version of Mitchell's classification of complex reflection groups in dimensions greater than four.
\end{abstract}

2000 Mathematics subject classification: 51F15, 20F55, 51M20.

Keywords and phrases: systems of lines, unitary reflection group, complex reflection group, pseudo-reflection.

\section{Introduction}

In a fundamental paper [2], Cameron, Goethals, Seidel and Shult consider sets of lines through the origin of $\mathbb{R}^{n}$ in which the angle between every pair of lines is $60^{\circ}$ or $90^{\circ}$. In this context a star is a set of three coplanar lines such that the angle between each pair is $60^{\circ}$. A set of lines through the origin is star-closed if for each pair of lines at $60^{\circ}$ the set contains the third line of the star; it is indecomposable if it cannot be written as the disjoint union of nonempty mutually orthogonal subsets. The indecomposable star-closed sets of lines in $\mathbb{R}^{n}$ correspond to the root systems of types $A_{n}, D_{n}, E_{6}, E_{7}$ and $E_{8}$ (see [2]). This result was a major tool in the determination of the graphs whose adjacency matrix has least eigenvalue -2 . Cvetković et al. give a complete account

(c) 2008 Australian Mathematical Society 1446-7887/08 \$A2.00+0.00 
in their book [5] and they determine the embeddings between Euclidean star-closed line systems. We extend these classification and embedding results to star-closed sets of lines in complex space $\mathbb{C}^{n}$. The main results are collected in Theorems 7.2, 7.3 and 9.1 .

The classification of star-closed line systems in $\mathbb{R}^{n}$ is equivalent to the well-known classification (see [1]) of Coxeter groups whose Dynkin diagram is 'simply laced'. The classification of star-closed line systems in $\mathbb{C}^{n}$ relates in a similar way to a theorem of Mitchell [9] on complex reflection groups in dimensions greater than four. Mitchell's results form an essential part of the Shephard and Todd [10] classification of primitive complex reflection groups. (See Kantor [7] for an overview.)

\section{Reflections and star-closed line systems}

The complex vector space $\mathbb{C}^{n}$ of dimension $n$ comes equipped with the standard hermitian inner product $(u, v)$ between $u=\left(u_{1}, u_{2}, \ldots, u_{n}\right)$ and $v=\left(v_{1}, v_{2}, \ldots, v_{n}\right)$ defined by $(u, v)=u_{1} \bar{v}_{1}+u_{2} \bar{v}_{2}+\cdots+u_{n} \bar{v}_{n}$. A linear transformation $\psi: \mathbb{C}^{n} \rightarrow \mathbb{C}^{n}$ is unitary if it preserves the inner product; that is, if $(u \psi, v \psi)=(u, v)$ for all $u, v \in \mathbb{C}^{n}$.

In this paper, a vector $u \in \mathbb{C}^{n}$ is a root if $(u, u)=2$. If $u$ is a root, $\langle u\rangle$ denotes the line spanned by $u$. Roots $u$ and $v$ (or the lines they span) are said to be at $60^{\circ}$ if $|(u, v)|=1$; they are at $90^{\circ}$ if $(u, v)=0$. This coincides with the usual notion in $\mathbb{R}^{n}$. A star consists of three coplanar lines through the origin such that each pair is at $60^{\circ}$. As in the Euclidean case, given two lines at $60^{\circ}$, there is a unique star containing them.

A set $S$ of lines through the origin of $\mathbb{C}^{n}$ is a line system if each pair of lines is at $60^{\circ}$ or $90^{\circ}$. The paper of Delsarte et al. [6] provides explicit bounds on the size of a line system. In particular, every line system is finite, and Koornwinder [8] gives a short, elegant proof of this finiteness result.

A line system $S$ is star-closed if for each pair of lines at $60^{\circ}$ the third line of the star belongs to $S$. Our immediate aim is to show that star-closed line systems correspond to certain finite groups generated by reflections of order two.

Given a root $a$, the complex reflection in the hyperplane $a^{\perp}$ perpendicular to $a$ is the unitary transformation $\rho_{a}$ defined by $v \rho_{a}=v-(v, a) a$.

We say that $a$ is a root of $\rho_{a}$ and note that $\rho_{a}=\rho_{b}$ if and only if $\langle a\rangle=\langle b\rangle$. The order of $\rho_{a}$ is two, but there are complex reflections of order greater than two acting on $\mathbb{C}^{n}$ (see [10]). The latter will not enter into our calculations.

LEMMA 2.1. Given roots $a$ and $b$ we have $\left|\rho_{a} \rho_{b}\right|=2$ if and only if $a$ and $b$ are at $90^{\circ}$ and $\left|\rho_{a} \rho_{b}\right|=3$ if and only if $a$ and $b$ are at $60^{\circ}$.

PROOF. We may suppose that $a$ and $b$ are linearly independent. The reflections $\rho_{a}$ and $\rho_{b}$ fix the subspace $\langle a, b\rangle$ and the matrices of the restrictions of $\rho_{a}$ and $\rho_{b}$ with respect to the basis $a, b$ are

$$
A=\left[\begin{array}{cc}
-1 & 0 \\
-(b, a) & 1
\end{array}\right] \quad \text { and } \quad B=\left[\begin{array}{cc}
1 & -(a, b) \\
0 & -1
\end{array}\right]
$$


Consequently,

$$
A B=\left[\begin{array}{cc}
-1 & (a, b) \\
-(b, a) & |(a, b)|^{2}-1
\end{array}\right]
$$

and the order $m$ of $\rho_{a} \rho_{b}$ is also the order of $A B$.

The eigenvalues of $A B$ are $\exp (2 \pi i h / m)$ and $\exp (-2 \pi i h / m)$ for some $h$ coprime to $m$, where $1 \leq h<m$. On taking the trace we see that $|(a, b)|^{2}-2=2 \cos (2 \pi h / m)$ and so $|(a, b)|=2|\cos (\pi h / m)|$. The result follows.

LEMMA 2.2. Given roots $a$ and $b$ at $60^{\circ}$, the root $c=b \rho_{a}=-(b, a) a \rho_{b}$ spans the third line of the star determined by $a$ and $b$. Furthermore, $\rho_{c}=\rho_{b} \rho_{a} \rho_{b}=\rho_{a} \rho_{b} \rho_{a}$.

PROOF. We have $b \rho_{a}=b-(b, a) a=-(b, a)(a-(a, b) b)=-(b, a) a \rho_{b}$. Furthermore, $\left(b \rho_{a}, b\right)=1$ and $\left(b \rho_{a}, a\right)=-(b, a)$, whence $b \rho_{a}$ is at $60^{\circ}$ to $a$ and $b$.

THEOREM 2.3. Given a line system $S$ in $\mathbb{C}^{n}$ and a set of roots $\Sigma$ of $S$, the order of $\rho_{a} \rho_{b}$ is one, two or three, for all $a, b \in \Sigma$. Furthermore, $S$ is star-closed if and only if $S \rho_{a}=S$ for all $a \in \Sigma$. Conversely, if $G$ is a group of unitary transformations of $\mathbb{C}^{n}$ such that the order of the product of every pair of reflections of order two in $G$ is at most three, then the set of lines spanned by the roots of these reflections is a star-closed line system.

PROOF. This is an immediate consequence of the preceding two lemmas.

A line system $S$ is decomposable if there is a partition of the set of roots into a pair of nonempty subsets $A$ and $B$ such that $(a, b)=0$ for all $a \in A, b \in B$. In this case we write $S=A \perp B$. The line system is indecomposable if it is not decomposable. We write $S=k T$ to indicate that $S$ is the orthogonal sum of $k$ copies of $T$.

Let $W(S)$ denote the group generated by the reflections $\rho_{a}$, where $a$ is a root of $S$. Every line system $S$ is the orthogonal sum $S=S_{1} \perp S_{2} \perp \cdots \perp S_{k}$ of indecomposable line systems $S_{i}$ and $S$ is star-closed if and only if every $S_{i}$ is star-closed. In this case the group $W(S)$ is the direct product of the groups $W\left(S_{i}\right)$.

THEOREM 2.4. A star-closed line system $S$ is indecomposable if and only if $W(S)$ acts transitively on the lines of $S$.

PROOF. Suppose that $\ell$ and $m$ are lines of $S$ with roots $a$ and $b$, respectively. If $(a, b) \neq 0$, the reflection whose root spans the third line of the star containing $a$ and $b$ takes $\ell$ to $m$. If $S$ is indecomposable, there are roots $a=a_{0}, a_{1}, \ldots, a_{k}=b$ such that $\left(a_{i-1}, a_{i}\right) \neq 0$ for $1 \leq i \leq k$. Thus there is an element of $W(S)$ taking $\ell$ to $m$ and hence $W(S)$ is transitive on $S$.

Conversely, suppose that $S=A \perp B$, where $A$ and $B$ are star-closed and nonempty. If $a$ is the root of an element of $A$, then $\rho_{a}$ fixes $A$ and $B$, and so $W(S)$ is not transitive on $S$. 
The considerations so far show that star-closed line systems correspond to groups generated by reflections of order two in which the order of the product of any two reflections is at most three. Consequently, the indecomposable line systems in $\mathbb{R}^{n}$ are the well-known root systems of types $A_{n}, D_{n}, E_{6}, E_{7}$ and $E_{8}$. In $\mathbb{C}^{n}$ they correspond to the complexification of these root systems and to the root systems of the imprimitive groups $G(3,3, n)$, or the primitive groups $G_{33}$ and $G_{34}$ in the Shephard and Todd notation. Mitchell [9] proved that for a primitive group acting on a space of dimension at least five, the reflections have order two and the order of the product of any two reflections is at most three.

We generalize the results of Cvetković et al. [5, Chapter 3] on one-line extensions of Euclidean line systems to the complex case. This leads to the classification of all starclosed line systems and thus provides an alternative proof of part of the classification of complex reflection groups.

\section{Extensions of line systems}

Given a star-closed line system $S$, the star-closure $X^{*}$ of a subset $X$ of $S$ is the intersection of all star-closed line systems in $S$ that contain $X$; it can be obtained from $X$ by successively adjoining the third line of the star of each pair of lines at $60^{\circ}$, and so $W\left(X^{*}\right)$ is generated by the reflections $\rho_{a}$ where $a$ runs through the roots of $X$. The star-closure of a set of roots is defined to be the star-closure of the lines that they span.

Every line system in $\mathbb{R}^{n}$ is a subset of a star-closed system [2, Lemma 2.3]. However, in $\mathbb{C}^{n}$ this is no longer true. For example, consider the star with roots $a=(1,-1,0), b=(0,1,-1)$ and $c=(-1,0,1)$. If $v=(1,0, \omega)$, where $\omega$ is a primitive cube root of unity, then the lines $\langle a\rangle,\langle b\rangle$ and $\langle v\rangle$ are pairwise at $60^{\circ}$ but they are not part of a star-closed line system because $\langle c\rangle$ and $\langle v\rangle$ are neither at $60^{\circ}$ nor at $90^{\circ}$.

Given star-closed line systems $S$ and $T$ in $\mathbb{C}^{n}$, we say that $T$ is a simple extension of $S$ if $T \neq S$ and if $T$ is the star-closure of $S \cup\{\ell\}$ for some line $\ell \in T$; in [5, p. 72] this is called a one-line extension of $S$. We say that $T$ is a minimal extension of $S$ if for all star-closed line systems $U$ such that $S \subseteq U \varsubsetneqq T$ we have $S=U$. There are examples of simple extensions that are not minimal in Section 7.

The dimension of the line system $S$ is defined to be the dimension of the linear span $\langle S\rangle$ of $S$.

Two line systems $S_{1}$ and $S_{2}$ are said to be equivalent if there is a unitary transformation $\psi:\left\langle S_{1}\right\rangle \rightarrow\left\langle S_{2}\right\rangle$ such that $S_{1} \psi=S_{2}$. In this case we write $S_{1} \simeq S_{2}$.

THEOREM 3.1. If $S$ and $T$ are indecomposable star-closed line systems, and $S \subset T$, then there is a sequence $S=S_{0} \subset S_{1} \subset \cdots \subset S_{k}=T$ of indecomposable star-closed line systems such that, for $1 \leq i \leq k, S_{i}$ is a simple extension of $S_{i-1}$.

Proof. Since $T$ is indecomposable, there is a line $\ell \in T$ that is neither in $S$ nor orthogonal to $S$. Let $S_{1}$ be the star-closure of $S \cup\{\ell\}$. The set $T$ is finite, $\left|T \backslash S_{1}\right|<$ $|T \backslash S|$, and thus the result follows by induction. 


\section{Line systems for imprimitive reflection groups}

For each positive integer $m$, let $U_{m}$ denote the group of all $m$ th roots of unity. Given a positive integer $n$ and a divisor $p$ of $m$, define $G(m, p, n)$ to be the semidirect product of the group of diagonal matrices

$$
A(m, p, n)=\left\{\operatorname{diag}\left(\theta_{1}, \theta_{2}, \ldots, \theta_{n}\right) \mid\left(\theta_{1} \theta_{2} \cdots \theta_{n}\right)^{m / p}=1, \theta_{i} \in U_{m}\right\}
$$

by the group $\operatorname{Sym}(n)$ of $n \times n$ permutation matrices.

Shephard and Todd [10] showed that every irreducible imprimitive complex reflection group is conjugate to $G(m, p, n)$ for some $m>1$. The reflections in $G(m, p, n)$ have order two and the order of the product of any two of them is at most three if and only if $m=p$ and $m \leq 3$.

For $n \geq 2$, let $\mathcal{D}_{n}^{(m)}$ be the line system for the group $G(m, m, n)$. If $e_{1}, e_{2}, \ldots, e_{n}$ is an orthonormal basis for $\mathbb{C}^{n}$, representatives for the roots of $\mathcal{D}_{n}^{(m)}$ are the $m\left(\begin{array}{l}n \\ 2\end{array}\right)$ vectors $e_{i}-\zeta e_{j}$, for $1 \leq i<j \leq n$, where $\zeta \in U_{m}$. In particular, the roots of $\mathcal{D}_{n}^{(2)}$ are the vectors $e_{i} \pm e_{j}$ for $1 \leq i<j \leq n$, and the roots of $\mathcal{D}_{n}^{(3)}$ are the vectors $e_{i}-e_{j}$, $e_{i}-\omega e_{j}$ and $e_{i}-\omega^{2} e_{j}$ for $1 \leq i<j \leq n$, where $\omega$ is a primitive cube root of unity.

If $m=1$, the subgroup $A(1,1, n)$ is trivial and $G(1,1, n)$ is the symmetric group $\operatorname{Sym}(n)$. The line system for $G(1,1, n)$ is denoted by $\mathcal{A}_{n-1}$, and its roots are the vectors $e_{i}-e_{j}$, where $i<j$. Thus a star is a line system of type $\mathcal{A}_{2}$.

The line systems $\mathcal{D}_{n}^{(2)}(n \geq 3), \mathcal{D}_{n}^{(3)}(n \geq 2)$ and $\mathcal{A}_{n}(n \geq 1)$ are indecomposable and star-closed; moreover, $\mathcal{A}_{n-1}$ is a subset of $\mathcal{D}_{n}^{(2)}$ and $\mathcal{D}_{n}^{(3)}$. In low dimensions some of these line systems are equivalent: $\mathcal{A}_{2} \simeq \mathcal{D}_{2}^{(3)}, \mathcal{A}_{3} \simeq \mathcal{D}_{3}^{(2)}$ and $\mathcal{D}_{2}^{(2)} \simeq 2 \mathcal{A}_{1}$.

The group $W\left(\mathcal{A}_{n-1}\right)=\operatorname{Sym}(n)$ acts transitively on the lines of $\mathcal{D}_{n}^{(2)}$ not in $\mathcal{A}_{n-1}$ and transitively on the lines of $\mathcal{D}_{n}^{(3)}$ not in $\mathcal{A}_{n-1}$. Thus $\mathcal{D}_{n}^{(2)}$ and $\mathcal{D}_{n}^{(3)}$ are minimal extensions of $\mathcal{A}_{n-1}$.

\section{Line systems for primitive reflection groups}

In addition to the line systems defined in the previous section there are just five other indecomposable star-closed line systems that we shall encounter: the line systems of types $E_{6}, E_{7}$ and $E_{8}$ in Euclidean space, and the complex line systems of types $K_{5}$ and $K_{6}$ discovered by Mitchell [9]. (The notation for the complex line systems was introduced by Cohen [3] and there are descriptions in the Atlas of Finite Groups [4, pp. 26, 52].)

The line system $\mathcal{E}_{8}$ is the union of $\mathcal{D}_{8}^{(2)}$ and the set $X$ of lines spanned by the 64 roots $\frac{1}{2}\left(e_{1} \pm e_{2} \pm \cdots \pm e_{8}\right)$, where there are an even number of positive coefficients. This line system is star-closed and contains 120 lines. The sets $\mathcal{D}_{8}^{(2)}$ and $X$ are the orbits of $W\left(\mathcal{D}_{8}^{(2)}\right)$ on $\mathcal{E}_{8}$.

The stabilizer of $z=\frac{1}{2}\left(e_{1}+e_{2}+\cdots+e_{8}\right)$ in $W\left(\mathcal{D}_{8}^{(2)}\right)$ is $\operatorname{Sym}(8)$ and this group has two orbits on the lines of $\mathcal{E}_{8}$ at $60^{\circ}$ to $z$ : the 28 lines of $\mathcal{D}_{8}^{(2)}$ with roots 
$e_{i}+e_{j}(1 \leq i<j \leq 8)$ and the 28 lines of $X$ whose roots have two or six negative coefficients. These orbits are interchanged by the action of $\rho_{z}$. It follows that $W\left(\mathcal{E}_{8}\right)$ acts transitively on the lines and stars of $\mathcal{E}_{8}$.

The line system $\mathcal{E}_{7}$ is the set of 63 lines of $\mathcal{E}_{8}$ orthogonal to $z$. Since $W\left(\mathcal{E}_{8}\right)$ is transitive on lines, if $\ell \in \mathcal{E}_{8}$ the lines of $\mathcal{E}_{8}$ orthogonal to $\ell$ form a star-closed line system equivalent to $\mathcal{E}_{7}$. Furthermore, $\mathcal{E}_{7}$ is indecomposable since $\mathcal{A}_{7} \subset \mathcal{E}_{7}$.

The line system $\mathcal{E}_{6}$ is the set of 36 lines of $\mathcal{E}_{8}$ orthogonal to the star with roots $e_{1}-e_{2}, e_{2}-e_{3}$ and $e_{1}-e_{3}$. The 15 lines of $\mathcal{E}_{6}$ orthogonal to $z$ form a subsystem equivalent to $\mathcal{A}_{5}$ and thus $\mathcal{A}_{5} \perp \mathcal{A}_{1} \subset \mathcal{E}_{6}$.

In order to describe the remaining examples it will be convenient to represent vectors by their coordinates with respect to the orthonormal basis $e_{1}, e_{2}, \ldots, e_{n}$ of $\mathbb{C}^{n}$.

Let $\omega$ denote the cube root of unity $\frac{1}{2}(-1+i \sqrt{3})$ and set $\theta=\omega-\omega^{2}=i \sqrt{3}$. The line system $\mathcal{K}_{6}$ is the union of $\mathcal{D}_{6}^{(3)}$ with the set of lines spanned by all the 81 roots $\theta^{-1}\left(1, \alpha_{2}, \ldots, \alpha_{6}\right)$, where $\alpha_{i}$ is a power of $\omega$ and $\prod_{i=2}^{6} \alpha_{i}=1$. These 126 lines form a star-closed line system in $\mathbb{C}^{6}$ and since $\mathcal{D}_{6}^{(3)} \subset \mathcal{K}_{6}$, it is indecomposable.

The line system $\mathcal{K}_{6}$ is equivalent to $\overline{\mathcal{K}}_{6}$, which is the union of $\mathcal{D}_{6}^{(2)}$ with the set of 96 lines spanned by the images of $\frac{1}{2}(1,1,1,1,1, \theta)$ under the action of $W\left(\mathcal{D}_{6}^{(2)}\right)$. To see this, let $J_{n}$ denote the $n \times n$ matrix in which every entry is 1 and let $U=$ $\left(\frac{1}{6} \omega \theta J_{6}+I\right) \operatorname{diag}(1,1,1,1,1, \omega)$. Then $U$ is a unitary matrix, and the map $v \mapsto v U$ is an equivalence between $\overline{\mathcal{K}}_{6}$ and $\mathcal{K}_{6}$. The vectors $e_{i}-e_{j}$, where $1 \leq i<j \leq 5$, are the roots of the line system $\mathcal{A}_{4}$ in both $\overline{\mathcal{K}}_{6}$ and $\mathcal{K}_{6}$. The transformation $U$ sends the subsystem $\mathcal{A}_{5}$ of $\overline{\mathcal{K}}_{6}$ to the star-closure in $\mathcal{K}_{6}$ of $\mathcal{A}_{4}$ with the line $\left\langle e_{5}-\omega e_{6}\right\rangle$.

Thus, in $\mathcal{K}_{6}$, the line system $\mathcal{A}_{4}$ is contained in three line systems of type $\mathcal{A}_{5}$, representing the orbits of $W\left(\mathcal{K}_{6}\right)$ on subsystems of type $\mathcal{A}_{5}$. They are obtained by taking the star closures of $\mathcal{A}_{4}$ with lines $\left\langle e_{5}-e_{6}\right\rangle,\left\langle e_{5}-\omega e_{6}\right\rangle$ and $\left\langle e_{5}-\omega^{2} e_{6}\right\rangle$, respectively.

The line system $\mathcal{K}_{5}$ is the subsystem of $\mathcal{K}_{6}$ orthogonal to $\theta^{-1}(1,1, \ldots, 1)$. The group $W\left(\mathcal{K}_{6}\right)$ is transitive on lines and thus for all $\ell \in \mathcal{K}_{6}$, the 45 lines of $\mathcal{K}_{6}$ orthogonal to $\ell$ form a line system equivalent to $\mathcal{K}_{5}$. In particular, since the lines of $\mathcal{D}_{4}^{(3)}$ are orthogonal to $e_{1}-e_{2}$, we see that $\mathcal{K}_{5}$ contains line systems of type $\mathcal{D}_{4}^{(3)}$.

It will be useful later to know that $\mathcal{K}_{5}$ is equivalent to the line system $\overline{\mathcal{K}}_{5}$ in $\mathbb{C}^{5}$ that is the union of the 12 lines of $\mathcal{D}_{4}^{(2)}$ (on the first four coordinates), the 32 lines spanned by the images of $\frac{1}{2}(1,1,1, i \sqrt{3}, \sqrt{2})$ under the action of $W\left(\mathcal{D}_{4}^{(2)}\right)$, and the line $\langle(0,0,0,0, \sqrt{2})\rangle$. The unitary transformation $\psi: \mathbb{C}^{5} \rightarrow \mathbb{C}^{6}$ such that $e_{i} \psi=e_{i}$ $(1 \leq i \leq 4)$ and $e_{5} \psi=(1 / \sqrt{2})\left(e_{5}+e_{6}\right)$ defines an equivalence between $\overline{\mathcal{K}}_{5}$ and the $\mathcal{K}_{5}$-subsystem of $\overline{\mathcal{K}}_{6}$ orthogonal to $e_{5}-e_{6}$.

In $\overline{\mathcal{K}}_{6}$, the 20 lines orthogonal to $e_{6}$ are the lines of $\mathcal{D}_{5}^{(2)}$. These lines together with the 16 images of $\left\langle\frac{1}{2}(1,1,1,1,1, \theta)\right\rangle$ under the action of $W\left(\mathcal{D}_{5}^{(2)}\right)$ form a line system equivalent to $\mathcal{E}_{6}$. The equivalence is given by the transformation $\varphi: \mathbb{C}^{6} \rightarrow \mathbb{C}^{8}$ where 
$e_{i} \varphi=e_{i}(1 \leq i \leq 5)$ and $e_{6} \varphi=\theta^{-1}\left(e_{6}+e_{7}+e_{8}\right)$.

In $\mathcal{K}_{6}$ the $\mathcal{A}_{5}$ subsystem which is the star-closure of $\mathcal{A}_{4}$ and $e_{5}-e_{6}$ is orthogonal to $\theta^{-1}(1,1, \ldots, 1)$ and thus lies in $\mathcal{K}_{5}$. However, the other $\mathcal{A}_{5}$ subsystems extending $\mathcal{A}_{4}$ are interchanged by complex conjugation and furthermore there is no line of $\mathcal{K}_{6}$ orthogonal to either of them.

Thus the first $\mathcal{A}_{5}$ subsystem has simple extensions to $\mathcal{A}_{5} \perp \mathcal{A}_{1}, \mathcal{E}_{6}, \mathcal{K}_{5}$ and $\mathcal{D}_{6}^{(3)}$ whereas the second and third subsystems have simple extensions to $\mathcal{A}_{6}, \mathcal{D}_{6}^{(2)}, \mathcal{D}_{6}^{(3)}$ and to $\mathcal{K}_{6}$ itself. The $\mathcal{A}_{6}$ extension is the star-closure of the lines $\left\langle e_{1}-e_{2}\right\rangle,\left\langle e_{2}-e_{3}\right\rangle$, $\left\langle e_{3}-e_{4}\right\rangle,\left\langle e_{4}-e_{5}\right\rangle,\left\langle e_{5}-\omega e_{6}\right\rangle$ and $\left\langle\theta^{-1}\left(e_{1}+e_{2}+\cdots+e_{6}\right)\right\rangle$.

\section{The Goethals-Seidel decomposition}

Throughout this section suppose that $S$ is an indecomposable star-closed line system in $\mathbb{C}^{n}$ and that $\Sigma$ is a set of roots for $S$. We refine the choice of $\Sigma$ as we proceed.

Assume that $S$ has at least two lines. We choose roots $a$ and $b$ corresponding to a pair of lines at $60^{\circ}$ and scale $b$ so that $(a, b)=-1$. Then $c=-a-b$ is a root that spans the third line of the star of $a$ and $b$ and we can suppose that $a, b$ and $c$ belong to $\Sigma$. Extending the notation of [2], the Goethals-Seidel decomposition has components:

$$
\begin{aligned}
\Gamma_{a} & =\{x \in \Sigma \mid(a, x)=0 \text { and }(b, x) \neq 0\}, \\
\Gamma_{b} & =\{x \in \Sigma \mid(b, x)=0 \text { and }(c, x) \neq 0\}, \\
\Gamma_{c} & =\{x \in \Sigma \mid(c, x)=0 \text { and }(a, x) \neq 0\}, \\
\Delta & =\{x \in \Sigma \mid(a, x)=0 \text { and }(b, x)=0\}, \quad \text { and } \\
\Lambda & =\{x \in \Sigma \backslash\{a, b, c\} \mid(a, x)(b, x)(c, x) \neq 0\} .
\end{aligned}
$$

It is easy to see that $\Sigma$ is the disjoint union of the sets $\{a, b, c\}, \Gamma_{a}, \Gamma_{b}, \Gamma_{c}, \Delta$ and $\Lambda$. Some of these sets may be empty; indeed, we shall see that $S$ is a Euclidean line system if and only if $\Lambda=\emptyset$. In $\mathcal{D}_{n}^{(3)}$ every line is in a unique star such that $\Gamma_{a}=\emptyset$.

Scale the roots $x \in \Gamma_{a}$ so that $(b, x)=1$, and hence $(c, x)=-1$. Similarly, scale $x \in \Gamma_{b}$ so that $(c, x)=1$, and scale $x \in \Gamma_{c}$ so that $(a, x)=1$.

LeMMA 6.1. $\Gamma_{b}=\Gamma_{a} \rho_{c}=\left\{x+c \mid x \in \Gamma_{a}\right\}$ and $\Gamma_{c}=\Gamma_{a} \rho_{b}=\left\{x-b \mid x \in \Gamma_{a}\right\}$.

PRoOF. If $x \in \Gamma_{a}$, then $(x, c)=-1$ and thus $x \rho_{c}=x+c$. Furthermore, $(b, x+c)$ $=0$ and $(c, x+c)=1$, hence $x+c \in \Gamma_{b}$. Similarly $x \rho_{b}=x-b \in \Gamma_{c}$. Conversely, for $y \in \Gamma_{b}$ we have $y \rho_{c}=y-c \in \Gamma_{a}$, and for $z \in \Gamma_{c}$ we have $z \rho_{b}=z+b$. Thus $\Gamma_{b}=\Gamma_{a} \rho_{c}$ and $\Gamma_{c}=\Gamma_{a} \rho_{b}$.

The following lemma shows that cube roots of unity enter into our calculations in an essential way.

LEMMA 6.2. Suppose that $\sigma$ is a star and that $\ell \notin \sigma$ is a line at $60^{\circ}$ to a line of $\sigma$ with root $a$. Let $v$ be the root of $\ell$ such that $(a, v)=1$ and let $V$ be the subspace spanned by $\sigma$ and $\ell$. 
(i) If $\ell$ is orthogonal to a line of $\sigma$ with root $b$, where $(a, b)=-1$, then there is an orthonormal basis of $V$ with respect to which $a, b$ and $v$ have coordinates $(1,-1,0),(0,1,-1)$ and $(0,-1,-1)$. In this case the star-closure of $\sigma$ and $\ell$ is $\mathcal{D}_{3}^{(2)}$.

(ii) If $\ell$ is at $60^{\circ}$ to all three lines $\langle a\rangle,\langle b\rangle$ and $\langle c\rangle$ of $\sigma$, where $a+b+c=0$ and $(a, b)=-1$, then $(b, v) \in\left\{\omega, \omega^{2}\right\}$ and there is an orthonormal basis of $V$ with respect to which $a, b$ and $v$ have coordinates $(1,-1,0),(0,1,-1)$ and $(0,-1,(b, v))$. In this case the star-closure of $\sigma$ and $\ell$ is $\mathcal{D}_{3}^{(3)}$.

PROOF. First choose a vector $e \in V$ orthogonal to $a$ and $b$ and such that $(e, e)=3$. Since $(a, b)=-1$ the vectors $\frac{1}{3}(e+2 a+b), \frac{1}{3}(e-a+b)$ and $\frac{1}{3}(e-a-2 b)$ form an orthonormal basis of $V$ with respect to which $a$ and $b$ have coordinates $(1,-1,0)$ and $(0,1,-1)$. If $v$ has coordinates $(\alpha, \beta, \gamma)$ with respect to this basis, then $\beta=\alpha-1$.

If $(b, v)=0$, then $\gamma=\beta=\alpha-1$. On the other hand, if $\ell$ is at $60^{\circ}$ to $b$ and $c$, and if $\theta=(b, v)$, then $|\theta|=|1+\theta|=1$ and hence $\theta$ is $\omega$ or $\omega^{2}$. In both cases it follows that $v$ has coordinates $(\alpha, \alpha-1, \alpha+\theta)$, where $\theta=-1$ if $(b, v)=0$. Since $(v, v)=2$ we have $3|\alpha|^{2}+(\bar{\theta}-1) \alpha+(\theta-1) \bar{\alpha}=0$. The matrix $I-\bar{\alpha}(1-\bar{\theta})^{-1} J$ is unitary, fixes $a$ and $b$ and takes $v$ to $(0,-1, \theta)$. Thus the star-closure of $\sigma$ and $\ell$ exists: in case (i) it is $\mathcal{D}_{3}^{(2)}$ whereas in case (ii) it is $\mathcal{D}_{3}^{(3)}$.

COROllary 6.3 (see [2]). If $S$ is a line system in $\mathbb{R}^{k}$, then $S$ is a subset of a starclosed line system, and every line of $S$ not in a given star $\sigma$ of $S$ is orthogonal to at least one line of $\sigma$.

PROOF. Case (ii) of the lemma cannot occur. Therefore, if $m$ and $n$ are lines of $S$ at $60^{\circ}$ and if $p$ is the third line of the star of $m$ and $n$ in $\mathbb{R}^{k}$, then any line $\ell \neq p$ of $S$ is at $60^{\circ}$ or $90^{\circ}$ to $p$. Thus $S \cup\{p\}$ is again a line system and we obtain a star-closed line system by successively adjoining the third line of the star of each pair of lines at $60^{\circ}$.

Scale the roots $v \in \Lambda$ so that $(a, v)=1$. By Lemma 6.2 (ii), $(b, v) \in\left\{\omega, \omega^{2}\right\}$ and the star-closure of $a, b$ and $v$ is equivalent to $\mathcal{D}_{3}^{(3)}$.

The results obtained so far allow us to determine all star-closed line systems in low dimensions.

THEOREM 6.4. Let $S$ be a star-closed line system of dimension at most three.

(i) If $\operatorname{dim} S=1$, then $S \simeq \mathcal{A}_{1}$ and $S$ consists of a single line.

(ii) If $\operatorname{dim} S=2$, then $S$ is equivalent to $\mathcal{D}_{2}^{(2)} \simeq 2 \mathcal{A}_{1}$ or to a star $\mathcal{D}_{2}^{(3)} \simeq \mathcal{A}_{2}$.

(iii) If $\operatorname{dim} S=3$, then $S$ is equivalent to $3 \mathcal{A}_{1}, \mathcal{A}_{1} \perp \mathcal{A}_{2}, \mathcal{A}_{3} \simeq \mathcal{D}_{3}^{(2)}$ or $\mathcal{D}_{3}^{(3)}$.

PRoOF. (i) and (ii). The result is clear when $\operatorname{dim} S=1$. In $\mathbb{C}^{2}$, there is a unique star containing a pair of lines at $60^{\circ}$ and a unique line orthogonal to a given line: thus $S$ is either a pair of orthogonal lines or a star. 
(iii) If $S$ is decomposable, then $S=\mathcal{A}_{1} \perp T$, where $T$ is a star-closed system of dimension two. In this case $S$ is equivalent to $3 \mathcal{A}_{1}$ or $\mathcal{A}_{1} \perp \mathcal{A}_{2}$.

If $S$ is indecomposable and a simple extension of $\mathcal{A}_{2}$, then by Lemma $6.2 S$ is equivalent to $\mathcal{D}_{3}^{(2)}$ or $\mathcal{D}_{3}^{(3)}$. The line system $\mathcal{A}_{3}$ is a simple extension of $\mathcal{A}_{2}$ and therefore it is equivalent to $\mathcal{D}_{3}^{(2)}$.

It follows from Theorem 3.1 that to complete the proof it is enough to show that neither $\mathcal{D}_{3}^{(2)}$ nor $\mathcal{D}_{3}^{(3)}$ have simple extensions in $\mathbb{C}^{3}$. By way of contradiction, suppose that $S$ contains a line system $T$ and a root $v=(\alpha, \beta, \gamma)$ such that $\langle v\rangle \notin T$, where $T$ is either $\mathcal{D}_{3}^{(2)}$ or $\mathcal{D}_{3}^{(3)}$. If $v$ is orthogonal to $a=(1,-1,0)$ and $b=(0,1,-1)$ then $\alpha=\beta=\gamma$ and on taking the inner product of $v$ with $(1,1,0)$ or $(1,-\omega, 0)$ we reach a contradiction. Thus we may suppose that $(a, v)=1$ and hence $\beta=\alpha-1$. If $(b, v)=0$, then $\gamma=\alpha-1$. If $\theta=(b, v) \neq 0$, then $|\theta|=|1+\theta|=1$ and hence $\theta$ is $\omega$ or $\omega^{2}$. Thus $v=(\alpha, \alpha-1, \alpha+\theta)$, where $\theta$ is $-1, \omega$ or $\omega^{2}$. As in Lemma 6.2 we have $3|\alpha|^{2}+(\bar{\theta}-1) \alpha+(\theta-1) \bar{\alpha}=0$.

If $T=\mathcal{D}_{3}^{(2)}$, then $d=(1,1,0)$ is a root of $T$, which is not orthogonal to $v$. Therefore $|(d, v)|=1$ and hence $2|\alpha|^{2}-\alpha-\bar{\alpha}=0$. Combined with the previous equation this implies that $\alpha$ is 0 or 1 . But then $S$ contains $\mathcal{D}_{3}^{(3)}$ and this is a contradiction since the lines $\langle(1,1,0)\rangle$ and $\langle(1,-\omega, 0)\rangle$ are neither at $60^{\circ}$ nor $90^{\circ}$.

If $T=\mathcal{D}_{3}^{(3)}$, then $(1,-\omega, 0)$ and $\left(1,-\omega^{2}, 0\right)$ are roots of $T$. If both these roots are at $60^{\circ}$ to $v$, then

$$
\begin{aligned}
& 3|\alpha|^{2}+(\omega-1) \alpha+\left(\omega^{2}-1\right) \bar{\alpha}=0 \quad \text { and } \\
& 3|\alpha|^{2}+\left(\omega^{2}-1\right) \alpha+(\omega-1) \bar{\alpha}=0 .
\end{aligned}
$$

On adding these equations we find that $2|\alpha|^{2}-\alpha-\bar{\alpha}=0$. As before $\alpha=0$ or $\alpha=1$ and again we arrive at a contradiction. The remaining possibility is that $v$ is orthogonal to exactly one of $(1,-\omega, 0)$ or $\left(1,-\omega^{2}, 0\right)$. But in neither case is there a solution for $\alpha$.

Lemma 6.5. Suppose that $x, y \in \Gamma_{a}$, where $x \neq y$, and set $\mu=(x, y)$. Then $\mu \in\left\{0,1,-\omega,-\omega^{2}\right\}$ and the vectors $a, b, x$ and $y$ are linearly independent.

(i) If $\mu=0$, then $z=b-c-x-y \in \Gamma_{a}$ and $(x, z)=(y, z)=0$. The star-closure of $a, b, x$ and $y$ is equivalent to $\mathcal{D}_{4}^{(2)}$. Furthermore, if $w \in \Gamma_{a}$, then

$$
(w, x)+(w, y)+(w, z)=2 .
$$

(ii) If $\mu=1$, then a multiple of $x-y$ belongs to $\Delta$. The star-closure of $a, b, x$ and y is equivalent to $\mathcal{A}_{4}$.

(iii) If $\mu=-\omega$ or $\mu=-\omega^{2}$, then $\mu x+\bar{\mu} y \in \Gamma_{a}$ and $a+b-\mu x-y \in \Lambda$. The starclosure of $a, b, x$ and $y$ is equivalent to $\mathcal{D}_{4}^{(3)}$. 
PROOF. It follows from Lemma 6.2 applied to $-y$ and the star of $c$ and $x$ that $\mu \in\left\{0,1,-\omega,-\omega^{2}\right\}$. The Gram matrix of the vectors $a, b, x$ and $y$ is

$$
\left(\begin{array}{cccc}
2 & -1 & 0 & 0 \\
-1 & 2 & 1 & 1 \\
0 & 1 & 2 & \mu \\
0 & 1 & \bar{\mu} & 2
\end{array}\right)
$$

and its determinant is $4+2(\mu+\bar{\mu})-3|\mu|^{2}$, which is never 0 . Therefore, $a, b, x$ and $y$ are linearly independent. In particular, the star-closure $T$ of $a, b, x$ and $y$ is a line system of dimension four.

The star-closure of the roots $r=(1,-1,0,0,0), s=(0,1,-1,0,0)$ and $t=(0,0,1,-1,0)$ is $\mathcal{A}_{3}$, represented as a line system in $\mathbb{C}^{5}$. The map $\varphi$ such that $r \varphi=a, s \varphi=b$ and $t \varphi=-x$ preserves inner products and extends to an isometry from $\mathcal{A}_{3}$ to the star-closure of $a, b$ and $x$. For each possibility for $\mu$ we define a vector $u \in \mathbb{C}^{5}$ such that $\varphi$ extends to an isometry between the star-closure of $r, s, t$ and $u$ and $T$.

(i) If $\mu=0$, put $u=(1,1,0,0,0)$ and define $u \varphi=y$. Then the star-closure of $r$, $s, t$ and $u$ is $\mathcal{D}_{4}^{(2)}$ and isometric to $T$. From Lemma 6.1 we have $x+c, y-b \in \Sigma$, and since $(x+c, y-b)=-1$ it follows that $x+c+y-b$ is a root. Taking $z$ equal to $b-c-x-y$ we find that $z \in \Gamma_{a}$ and $(x, z)=(y, z)=0$. Thus, $x+y+z=b-c$ and so $(w, x)+(w, y)+(w, z)=2$ for all $w \in \Gamma_{a}$.

(ii) If $\mu=1$, put $u=(0,0,1,0,-1)$ and define $u \varphi=-y$. The star-closure of $r, s$, $t$ and $u$ is $\mathcal{A}_{4}$ and isometric to $T$. Furthermore $(0,0,0,1,-1) \varphi=x-y$ and so $x-y$ is a root of $S$. Since $(a, x-y)=(b, x-y)=0$, a multiple of $x-y$ belongs to $\Delta$.

(iii) If $\mu$ is $-\omega$ or $-\omega^{2}$, put $u=(0,0,1, \mu, 0)$ and define $u \varphi=-y$. Then the starclosure of $r, s, t$ and $u$ is $\mathcal{D}_{4}^{(3)}$ and isometric to $T$. Then $x-\mu y$ is a root of $S$ spanning the third line of the star of $x$ and $y$. Its multiple $z=\mu x+\bar{\mu} y$ satisfies $(a, z)=0$ and $(b, z)=1$ and thus $z \in \Gamma_{a}$. Since $y+c \in \Gamma_{b}$ and $(x, y+c)=-\bar{\mu}$ we see that $x+\bar{\mu}(y+c)$ is a root of $S$. If $v=-\mu x-y-c$, then $(a, v)=1,(b, v)=-\bar{\mu}$ and $(c, v)=-\mu$; hence $v \in \Lambda$.

\section{Extensions of line systems of type $\mathcal{D}_{n}^{(2)}$ and $\mathcal{D}_{n}^{(3)}$}

In preparation for the theorems of this section we first characterize the extensions $\mathcal{D}_{n}^{(k)} \subset \mathcal{D}_{n+1}^{(k)}$, where $k$ is 2 or 3 . To this end, let $V=\mathbb{C}^{n+1}$ and let $e_{1}, e_{2}, \ldots, e_{n+1}$ be an orthonormal basis of $V$. For the roots of $\mathcal{D}_{n}^{(k)}$ we take the vectors $e_{i}-\zeta e_{j}$, where $\zeta$ is a $k$ th root of unity and $1 \leq i<j \leq n$.

If $S$ is a simple extension of $\mathcal{D}_{n}^{(k)}$, we may suppose that $S$ is a line system in $V$ and that $S$ is the star-closure of $\mathcal{D}_{n}^{(k)}$ and a line $\ell$ with root $x$. We write $x=\left(\alpha_{1}, \alpha_{2}, \ldots, \alpha_{n+1}\right)$ using coordinates with respect to the basis $e_{1}, e_{2}, \ldots, e_{n+1}$.

LEMMA 7.1. Suppose that $S$ is the star-closure in $V$ of $\mathcal{D}_{n}^{(k)}$ and a line $\ell$ with root $x=\left(\alpha_{1}, \alpha_{2}, \ldots, \alpha_{n+1}\right)$ as above. If $S$ is indecomposable and if $\alpha_{i}=0$ for some $i \leq n$, then $S$ is equivalent to $\mathcal{D}_{n+1}^{(k)}$. 
ProOF. The group $G(k, 1, n)$ acts on $\mathbb{C}^{n+1}$ by permuting the first $n$ coordinates and multiplying their values by $k$ th roots of unity, thus fixing the line system $\mathcal{D}_{n}^{(k)}$. In particular, we may suppose that $\alpha_{1}=0$ and since $S$ is indecomposable we have $\alpha_{j} \neq 0$ for some $j \leq n$.

On taking inner products with $e_{1}-e_{i}$ we see that for $i \leq n$, either $\alpha_{i}=0$ or $\left|\alpha_{i}\right|=1$. Since $(x, x)=2$ it follows that $\alpha_{i} \neq 0$ for exactly two values of $i$. If these values are $i$ and $j$ with $i<j$, we scale $x$ so that $\alpha_{i}=1$. If $j \leq n$ and if $\zeta$ is a $k$ th root of unity, then $\left(x, e_{i}-\zeta e_{j}\right)=1-\alpha_{j} \bar{\zeta}$. If $k=2$ and $\alpha_{j} \neq \pm 1$, then $\left|1-\alpha_{j}\right|=\left|1+\alpha_{j}\right|=1$, which is a contradiction. If $k=3$, then $\alpha_{j} \neq 1$ and hence $\left|1-\alpha_{j}\right|=1$. It follows that $\alpha_{j}$ is $-\omega$ or $-\omega^{2}$ and thus $\ell \in \mathcal{D}_{n}^{(3)}$, contrary to the choice of $\ell$.

The only possibility is that $j=n+1$ and $\left|\alpha_{n+1}\right|=1$. The $(n+1) \times(n+1)$ diagonal matrix $\operatorname{diag}\left(1,1, \ldots, 1,-\alpha_{n+1}^{-1}\right)$ is unitary; it fixes every root of $\mathcal{D}_{n}^{(k)}$ and sends $x$ to $e_{i}-e_{n+1}$. Thus $S$ is equivalent to $\mathcal{D}_{n+1}^{(k)}$.

THEOREM 7.2. If $S$ is an indecomposable star-closed line system and a simple extension of $\mathcal{D}_{n}^{(2)}$ for $n \geq 3$, then the extension is equivalent to one of:

$$
\begin{array}{ccc}
\mathcal{D}_{n}^{(2)} \subset \mathcal{D}_{n+1}^{(2)}, & \mathcal{D}_{3}^{(2)} \subset \mathcal{A}_{4}, \quad \mathcal{D}_{3}^{(2)} \subset \mathcal{D}_{4}^{(3)}, \quad \mathcal{D}_{4}^{(2)} \subset \mathcal{K}_{5}, \quad \mathcal{D}_{5}^{(2)} \subset \mathcal{E}_{6}, \\
\mathcal{D}_{5}^{(2)} \subset \mathcal{K}_{6}, \quad \mathcal{D}_{6}^{(2)} \subset \mathcal{K}_{6}, \quad \mathcal{D}_{6}^{(2)} \subset \mathcal{E}_{7}, \quad \mathcal{D}_{7}^{(2)} \subset \mathcal{E}_{8} \quad \text { or } \quad \mathcal{D}_{8}^{(2)} \subset \mathcal{E}_{8} .
\end{array}
$$

These extensions are minimal except that

$$
\begin{gathered}
\mathcal{D}_{4}^{(2)} \subset \mathcal{D}_{4}^{(2)} \perp \mathcal{A}_{1} \subset \mathcal{K}_{5}, \quad \mathcal{D}_{5}^{(2)} \subset \mathcal{D}_{6}^{(2)} \subset \mathcal{K}_{6}, \quad \mathcal{D}_{5}^{(2)} \subset \mathcal{E}_{6} \subset \mathcal{K}_{6}, \\
\mathcal{D}_{6}^{(2)} \subset \mathcal{D}_{6}^{(2)} \perp \mathcal{A}_{1} \subset \mathcal{E}_{7} \quad \text { and } \mathcal{D}_{7}^{(2)} \subset \mathcal{D}_{8}^{(2)} \subset \mathcal{E}_{8}
\end{gathered}
$$

PROOF. In the notation introduced above we may suppose that $S$ is a line system in $\mathbb{C}^{n+1}$ and that $S$ is the star-closure of $\mathcal{D}_{n}^{(2)}$ and a line $\ell$ with root $x=\left(\alpha_{1}, \alpha_{2}, \ldots, \alpha_{n+1}\right)$. From Lemma 7.1 we may suppose that $\alpha_{i} \neq 0$ for all $i \leq n$.

Suppose that for some $i, j, k \leq n$, the six quantities $\pm \alpha_{i}, \pm \alpha_{j}$ and $\pm \alpha_{k}$ are distinct. On taking inner products with the roots $e_{i} \pm e_{j}$ we find that $\left|\alpha_{i}-\alpha_{j}\right|=\left|\alpha_{i}+\alpha_{j}\right|=1$. From this and the corresponding calculations with $e_{i} \pm e_{k}$ and $e_{j} \pm e_{k}$ it follows that

$$
\begin{aligned}
\left|\alpha_{i}\right|^{2} \pm\left(\bar{\alpha}_{i} \alpha_{j}+\alpha_{i} \bar{\alpha}_{j}\right)+\left|\alpha_{j}\right|^{2} & =1, \\
\left|\alpha_{i}\right|^{2} \pm\left(\bar{\alpha}_{i} \alpha_{k}+\alpha_{i} \bar{\alpha}_{k}\right)+\left|\alpha_{k}\right|^{2} & =1, \quad \text { and } \\
\left|\alpha_{j}\right|^{2} \pm\left(\bar{\alpha}_{j} \alpha_{k}+\alpha_{j} \bar{\alpha}_{k}\right)+\left|\alpha_{k}\right|^{2} & =1 .
\end{aligned}
$$

Thus $\left|\alpha_{i}\right|^{2}=\left|\alpha_{j}\right|^{2}=\left|\alpha_{k}\right|^{2}=(1 / 2)$. Putting $\theta=\alpha_{i} \bar{\alpha}_{j}$ we find that $\theta+\bar{\theta}=0$ and $\theta \bar{\theta}=(1 / 4)$, whence $\theta= \pm(1 / 2) i$. Thus $\alpha_{i}= \pm i \alpha_{j}$ and similarly $\alpha_{i}= \pm i \alpha_{k}$. But then $\alpha_{j}= \pm \alpha_{k}$, which is a contradiction.

We have shown that there exist $\alpha \neq \pm \beta$ such that $\alpha_{i} \in\{\alpha,-\alpha, \beta,-\beta\}$ for all $i \leq n$. On replacing $x$ by an image under $G(2,1, n)$ we may suppose that for all $i \leq n, \alpha_{i}$ is 
either $\alpha$ or $\beta$. If there are $k$ values of $i$ such that $\alpha_{i}=\alpha$, then without loss of generality $k \geq n-k$. Since $n \geq 3$ we have $k \geq 2$ and therefore $|\alpha|=\frac{1}{2}$.

Suppose at first that $k \neq n$. Then $|\alpha+\beta|=|\alpha-\beta|=1$ and consequently we have $|\alpha|^{2} \pm(\bar{\alpha} \beta+\alpha \bar{\beta})+|\beta|^{2}=1$. It follows that $|\alpha|^{2}+|\beta|^{2}=1, \bar{\alpha} \beta+\alpha \bar{\beta}=0$ and therefore $|\beta|^{2}=\frac{3}{4}$. If $n-k \geq 2$, then $|\beta|=\frac{1}{2}$, which is a contradiction. Thus $n=k+1$ and $\beta= \pm i \sqrt{3} \alpha$.

We scale $x$ so that $\alpha=\frac{1}{2}$ and $\beta=\frac{1}{2} i \sqrt{3}$. Thus

$$
x=\frac{1}{2}\left(1,1, \ldots, 1, i \sqrt{3}, 2 \alpha_{n+1}\right)
$$

and it follows that $2=(x, x)=\frac{1}{4} k+\frac{3}{4}+\left|\alpha_{n+1}\right|^{2}$. That is, $k+4\left|\alpha_{n+1}\right|^{2}=5$ and hence $k \leq 5$. We consider each possibility for $k$ in turn, noting that multiplying the last coordinate of $x$ by a root of unity does not change the equivalence class of $S$.

The case $k=2, n=3$. We have $\left|\alpha_{n+1}\right|=\frac{1}{2} \sqrt{3}$ and we take

$$
x=-\frac{1}{2} \omega^{2}(1,1, i \sqrt{3}, i \sqrt{3}) .
$$

If $y=(1,1,0,0)$, then $(x, y)=-\omega^{2}$ and so from Lemma 6.5 with $a=(1,-1,0,0)$ and $b=(0,1,-1,0)$ it follows that $S$ is equivalent to $\mathcal{D}_{4}^{(3)}$.

The case $k=3, n=4$. We have $\left|\alpha_{n+1}\right|=1 / \sqrt{2}$ and so without loss of generality $x=\frac{1}{2}(1,1,1, i \sqrt{3}, \sqrt{2})$. It follows that $S$ is the line system $\overline{\mathcal{K}}_{5} \simeq \mathcal{K}_{5}$ described in Section 5. In particular, $(0,0,0,0, \sqrt{2})$ is a root of $S$ and so $\mathcal{D}_{4}^{(2)} \perp \mathcal{A}_{1} \subset S$.

The case $k=4, n=5$. We have $\left|\alpha_{n+1}\right|=\frac{1}{2}$ and we take

$$
x=\frac{1}{2}(1,1,1,1, i \sqrt{3}, 1) .
$$

The vectors $x$ and $\frac{1}{2}(-1,-1,-1,1,-i \sqrt{3}, 1)$ are roots of $S$ and the third line of their star is spanned by $(0,0,0,1,0,1)$. The star-closure of this vector with $\mathcal{D}_{5}^{(2)}$ is $\mathcal{D}_{6}^{(2)}$. It follows that $S$ is the line system $\overline{\mathcal{K}}_{6}$ defined in Section 5 and thus $S$ is equivalent to $\mathcal{K}_{6}$. We have $\mathcal{D}_{5}^{(2)} \subset \mathcal{D}_{6}^{(2)} \subset \overline{\mathcal{K}}_{6}$ and so the extension is simple but not minimal.

As shown in Section 5, $\mathcal{D}_{5}^{(2)} \subset \mathcal{E}_{6} \subset \overline{\mathcal{K}}_{6}$. The group $W\left(\mathcal{D}_{5}^{(2)}\right)=G(2,2,5)$ has three orbits on the roots of $\overline{\mathcal{K}}_{6}$ not in $\mathcal{D}_{5}^{(2)}$. Their lengths are 10,16 and 80, and their starclosures with $\mathcal{D}_{5}^{(2)}$ are equivalent to the line systems $\mathcal{D}_{6}^{(2)}, \mathcal{E}_{6}$ and $\mathcal{K}_{6}$.

The case $k=5, n=6$. In this case $\alpha_{n+1}=0$ and therefore we regard $S$ as a line system in $\mathbb{C}^{6}$. That is, we take $x=\frac{1}{2}(1,1,1,1,1, i \sqrt{3})$. As in the previous case, $S$ is the line system $\overline{\mathcal{K}}_{6}$, which is equivalent to $\mathcal{K}_{6}$. 
The case $k=n$. In this case $k / 4+\left|\alpha_{n+1}\right|^{2}=2$ and so $k \leq 8$. Therefore we may take $x=\frac{1}{2}(1,1, \ldots, 1, \sqrt{8-k})$, and since all coordinate values are real, the results of [5, Theorem 3.3.3] apply. In particular, we obtain the extensions

$$
\begin{array}{lll}
\mathcal{D}_{3}^{(2)} \subset \mathcal{A}_{4}, & \mathcal{D}_{4}^{(2)} \subset \mathcal{D}_{5}^{(2)}, & \mathcal{D}_{5}^{(2)} \subset \mathcal{E}_{6}, \\
\mathcal{D}_{6}^{(2)} \subset \mathcal{E}_{7}, & \mathcal{D}_{7}^{(2)} \subset \mathcal{E}_{8} \quad \text { and } & \mathcal{D}_{8}^{(2)} \subset \mathcal{E}_{8} .
\end{array}
$$

These extensions are minimal except for $\mathcal{D}_{6}^{(2)} \subset \mathcal{E}_{7}$ and $\mathcal{D}_{7}^{(2)} \subset \mathcal{E}_{8}$, where we have $\mathcal{D}_{6}^{(2)} \subset \mathcal{D}_{6}^{(2)} \perp \mathcal{A}_{1} \subset \mathcal{E}_{7}$ and $\mathcal{D}_{7}^{(2)} \subset \mathcal{D}_{8}^{(2)} \subset \mathcal{E}_{8}$.

THEOREM 7.3. If $S$ is an indecomposable star-closed line system and a simple extension of $\mathcal{D}_{n}^{(3)}$, where $n \geq 2$, then the extension is equivalent to one of:

$$
\mathcal{D}_{n}^{(3)} \subset \mathcal{D}_{n+1}^{(3)}, \quad \mathcal{D}_{2}^{(3)} \subset \mathcal{D}_{3}^{(2)}, \quad \mathcal{D}_{4}^{(3)} \subset \mathcal{K}_{5}, \quad \mathcal{D}_{5}^{(3)} \subset \mathcal{K}_{6} \quad \text { or } \quad \mathcal{D}_{6}^{(3)} \subset \mathcal{K}_{6}
$$

The extensions are minimal except for $\mathcal{D}_{5}^{(3)} \subset \mathcal{D}_{6}^{(3)} \subset \mathcal{K}_{6}$.

PROOF. We may suppose that $S$ is the star-closure in $\mathbb{C}^{n+1}$ of $\mathcal{D}_{n}^{(3)}$ and a line $\ell$ with root $x=\left(\alpha_{1}, \alpha_{2}, \ldots, \alpha_{n+1}\right)$. Furthermore, by Lemma 7.1, we may suppose that $\alpha_{i} \neq 0$ for all $i \leq n$.

If, for $i, j \leq n$, none of $\alpha_{i}-\alpha_{j}, \alpha_{i}-\omega \alpha_{j}, \alpha_{i}-\omega^{2} \alpha_{j}$ are 0 , then

$$
\begin{aligned}
\left|\alpha_{i}\right|^{2}-\alpha_{i} \bar{\alpha}_{j}-\bar{\alpha}_{i} \alpha_{j}+\left|\alpha_{j}\right|^{2} & =1, \\
\left|\alpha_{i}\right|^{2}-\omega^{2} \alpha_{i} \bar{\alpha}_{j}-\omega \bar{\alpha}_{i} \alpha_{j}+\left|\alpha_{j}\right|^{2} & =1, \text { and } \\
\left|\alpha_{i}\right|^{2}-\omega \alpha_{i} \bar{\alpha}_{j}-\omega^{2} \bar{\alpha}_{i} \alpha_{j}+\left|\alpha_{j}\right|^{2} & =1 .
\end{aligned}
$$

Therefore, $\left|\alpha_{i}\right|^{2}+\left|\alpha_{j}\right|^{2}=1$ and hence $\alpha_{i} \bar{\alpha}_{j}+\bar{\alpha}_{i} \alpha_{j}=\omega^{2} \alpha_{i} \bar{\alpha}_{j}+\omega \bar{\alpha}_{i} \alpha_{j}=0$. Consequently $\alpha_{i} \bar{\alpha}_{j}=0$, contrary to assumption. Thus for all $j \leq n$ we find that $\alpha_{j}$ is equal to $\alpha_{1}, \omega \alpha_{1}$ or $\omega^{2} \alpha_{1}$. We can now transform $x$ so that $\alpha_{1}=\alpha_{2}=\cdots=\alpha_{n}$. Taking the inner product with $e_{1}-\omega e_{2}$ we find that $\left|\alpha_{1}-\omega^{2} \alpha_{1}\right|=1$ and hence $\left|\alpha_{j}\right|=1 / \sqrt{3}$ for all $j \leq n$.

We have $(x, x)=\frac{1}{3} n+\left|\alpha_{n+1}\right|^{2}=2$ and therefore $n \leq 6$. In the following, we let $\theta=i \sqrt{3}=\omega-\omega^{2}$.

The case $n=2$. It follows from Lemma 6.2 with $a=\left(\omega,-\omega^{2}, 0\right), b=(1,-1,0)$ and $v=x=-\theta^{-1}(1,1,2)$ that $S$ is equivalent to $\mathcal{D}_{3}^{(2)}$.

The case $n=3$. We have $\left|\alpha_{n+1}\right|=1$ and we may scale $x$ so that $x=-\theta^{-1}(1,1,1, \theta)$. The group $G(3,3,3)$ acts on $S$ and so $y=-\theta^{-1}(1,1,1, \omega \theta)$ is a root of $S$ such that $(x, y)=-\omega$. Thus from Lemma 6.5 with $a=(1,-1,0,0)$ and $b=\left(\omega,-\omega^{2}, 0,0\right)$ it follows that $S$ is equivalent to $\mathcal{D}_{4}^{(3)}$.

The case $n=4$. We have $\left|\alpha_{n+1}\right|^{2}=\frac{2}{3}$ and we may certainly suppose that $x=\theta^{-1}(1,1,1,1, \sqrt{2})$. The linear transformation $\varphi: \mathbb{C}^{5} \rightarrow \mathbb{C}^{6}$ given by $e_{i} \varphi=e_{i}$ $(1 \leq i \leq 4)$ and $e_{5} \varphi=(1 / \sqrt{2})\left(e_{5}+e_{6}\right)$ defines an equivalence between $S$ and the $\mathcal{K}_{5}$ subsystem of $\mathcal{K}_{6}$ orthogonal to $e_{5}-e_{6}$ (see Section 5 ). 
The case $n=5$. In this case $\left|\alpha_{n+1}\right|^{2}=\frac{1}{3}$ and therefore we may suppose that $x=\theta^{-1}(1,1,1,1,1,1)$. The group $G(3,3,5)$ acts on $S$ and therefore the element $y=\theta^{-1}\left(\omega, \omega, \omega, \omega, \omega^{2}, 1\right)$ is a root of $S$. The third line of the star of $x$ and $y$ is spanned by $z=\left(0,0,0,0,1,-\omega^{2}\right)$. The star-closure of $\mathcal{D}_{5}^{(3)}$ and $z$ is $\mathcal{D}_{6}^{(3)}$. Thus $S$ is the line system $\mathcal{K}_{6}$ defined in Section 5 and the extension is simple but not minimal.

The case $n=6$. In this case $\alpha_{n+1}=0$. Therefore we may suppose that $S$ is a line system in $\mathbb{C}^{6}$ and that $x=\theta^{-1}(1,1,1,1,1,1)$. As in the previous case we have $S=\mathcal{K}_{6}$.

All extensions except $\mathcal{D}_{5}^{(3)} \subset \mathcal{D}_{6}^{(3)} \subset \mathcal{K}_{6}$ are minimal.

\section{Further structure of line systems in $\mathbb{C}^{n}$}

Throughout this section let $S$ be an indecomposable star-closed line system in $\mathbb{C}^{n}$ and let $\Sigma$ be a set of roots for $S$. As in Section 6 choose roots $a$ and $b$ corresponding to a pair of lines at $60^{\circ}$ and scale $b$ so that $(a, b)=-1$. Put $c=-a-b$ and define $\Gamma_{a}, \Gamma_{b}, \Gamma_{c}, \Delta$ and $\Lambda$ as before.

LEMMA 8.1. If $\Gamma_{a} \neq \emptyset$, then $\left\{\langle x-y\rangle \mid x, y \in \Gamma_{a}\right.$ and $\left.(x, y)=1\right\}$ is the set of lines spanned by the elements of $\Delta$.

PRoOF. If $x, y \in \Gamma_{a}$ and $(x, y)=1$, then $x-y$ is a root of $S$ and a multiple belongs to $\Delta$.

To prove the converse we extend the argument of [2]. Suppose that $z \in \Delta$ and that there exists $x \in \Gamma_{a}$ such that $(z, x) \neq 0$. Scale $z$ so that $(z, x)=-1$. Then $x+z$ is a root of $S$ and $z+x \in \Gamma_{a}$ because $(a, x+z)=0$ and $(b, x+z)=1$. Furthermore $(x+z, x)=1$ and $z=(x+z)-x$. To complete the proof suppose that $\Delta^{\prime} \neq \emptyset$, where

$$
\Delta^{\prime}=\left\{z \in \Delta \mid(z, x)=0 \text { for all } x \in \Gamma_{a}\right\} .
$$

We show that this leads to a contradiction. The roots $a, b, c$ and, by Lemma 6.1, the elements of $\Gamma_{a}, \Gamma_{b}$ and $\Gamma_{c}$ are orthogonal to every element of $\Delta^{\prime}$. Moreover, we have just shown that every element of $\Delta \backslash \Delta^{\prime}$ is a multiple of the difference of two elements of $\Gamma_{a}$ and therefore orthogonal to every element of $\Delta^{\prime}$. Since $S$ is indecomposable it follows that there exists $s \in \Lambda$ and $t \in \Delta^{\prime}$ such that $(s, t) \neq 0$.

By Lemma 6.2 (ii) the star-closure of $a, b$ and $s$ is equivalent to $\mathcal{D}_{3}^{(3)}$ and by Theorem 7.3 the star-closure $T$ of $a, b, s$ and $t$ is equivalent to $\mathcal{D}_{4}^{(3)}$. Since $t$ is orthogonal to $a$ and $b$ we may suppose that $a$ and $b$ correspond to $(1,-1,0,0)$ and $(1,-\omega, 0,0)$, respectively. If $u \in \Gamma_{a}$, it follows from Theorem 7.3 that the star-closure of $T$ and $u$ is $\mathcal{D}_{5}^{(3)}$ or $\mathcal{K}_{5}$. But in $\mathcal{D}_{5}^{(3)}$ we have $\Gamma_{a}=\emptyset$ and in $\mathcal{K}_{5}$ there is an element of $\Gamma_{a}$ not orthogonal to $t$. This contradiction completes the proof.

COROLlary 8.2. If $\Gamma_{a} \neq \emptyset$, the roots of $S$ can be normalized so that every root is a linear combination of elements of $\{a, b, c\} \cup \Gamma_{a}$ with coefficients from $\left\{ \pm 1, \pm \omega, \pm \omega^{2}\right\}$. 
PROOF. From Lemmas 6.1 and 6.2 the result holds for the elements of $\Gamma_{b}, \Gamma_{c}$ and $\Delta$. If $s \in \Lambda$ and $x \in \Gamma_{a}$ it follows from Lemma 6.2 (ii) and Theorem 7.3 that the starclosure $T$ of $a, b, x$ and $s$ is $\mathcal{D}_{4}^{(3)}$. In $\mathcal{D}_{4}^{(3)}$ we may suppose that $a=(1,-1,0,0)$, $b=(0,1,-1,0)$ and $x=(0,0,-1,1)$. If $y=(0,0,-1, \omega)$, then $T$ is the starclosure of $a, b, x$ and $y$. We have $y \in \Gamma_{a}$ and $s$ is a linear combination of $a, b, x$ and $y$ with coefficients from $\left\{ \pm 1, \pm \omega, \pm \omega^{2}\right\}$.

THEOREM 8.3. If $\Gamma_{a}=\emptyset$, then $S$ is $\mathcal{D}_{n}^{(3)}$ for some $n$.

ProOf. $S$ is indecomposable hence $\Lambda \neq \emptyset$, otherwise $S=\{a, b, c\} \perp \Delta$. If $s \in \Lambda$, the star-closure of $a, b$ and $s$ is $\mathcal{D}_{3}^{(3)}$ and it follows from Theorems 3.1 and 7.3 that $S$ is $\mathcal{D}_{n}^{(3)}$ or that $S$ contains $\mathcal{K}_{5}$ or $\mathcal{K}_{6}$. However, if $S$ contains $\mathcal{K}_{5}$ or $\mathcal{K}_{6}$, then $\Gamma_{a} \neq \emptyset$.

We are now able to characterize the Euclidean line systems.

THEOREM 8.4. The line system $S$ is the complexification of a Euclidean line system if and only if $\Lambda=\emptyset$.

PROOF. If $S$ is Euclidean, it follows from Lemma 6.2 (ii) that $\Lambda=\emptyset$.

Conversely, if $\Lambda=\emptyset$, then $(x, y) \in\{0,1\}$ for all $x \neq y \in \Gamma_{a}$. But the roots $a, b$ together with $\Gamma_{a}$ generate the entire root system and therefore the inner product of every pair of roots is real. It follows that $S$ is the complexification of a Euclidean root system.

\section{Extensions of line systems of types $\mathcal{A}_{n}, \mathcal{E}_{n}$ and $\mathcal{K}_{n}$}

THEOREM 9.1. Suppose that $S$ is an indecomposable star-closed line system and a simple extension of $T$, where $T$ is one of $\mathcal{A}_{n}(n \geq 2), \mathcal{E}_{n}(n=6,7,8)$ or $\mathcal{K}_{n}(n=5,6)$. Then the extension $T \subset S$ is equivalent to one of:

$$
\begin{gathered}
\mathcal{A}_{n} \subset \mathcal{A}_{n+1}, \quad \mathcal{A}_{n} \subset \mathcal{D}_{n+1}^{(2)}, \quad \mathcal{A}_{n} \subset \mathcal{D}_{n+1}^{(3)}, \\
\mathcal{A}_{4} \subset \mathcal{K}_{5}, \quad \mathcal{A}_{5} \subset \mathcal{K}_{5}, \quad \mathcal{A}_{5} \subset \mathcal{E}_{6}, \quad \mathcal{A}_{5} \subset \mathcal{K}_{6}, \\
\mathcal{A}_{6} \subset \mathcal{K}_{6}, \quad \mathcal{A}_{6} \subset \mathcal{E}_{7}, \\
\mathcal{A}_{7} \subset \mathcal{E}_{7}, \quad \mathcal{A}_{7} \subset \mathcal{E}_{8}, \quad \mathcal{A}_{8} \subset \mathcal{E}_{8}, \\
\mathcal{E}_{6} \subset \mathcal{E}_{7}, \quad \mathcal{E}_{6} \subset \mathcal{K}_{6}, \quad \mathcal{E}_{7} \subset \mathcal{E}_{8} \quad \text { or } \quad \mathcal{K}_{5} \subset \mathcal{K}_{6} .
\end{gathered}
$$

The extensions are minimal except that for suitable choices of $\mathcal{A}_{5}$ subsystems we have

$$
\begin{aligned}
& \mathcal{A}_{4} \subset \mathcal{A}_{5} \subset \mathcal{K}_{5}, \\
& \mathcal{A}_{5} \subset \mathcal{A}_{5} \perp \mathcal{A}_{1} \subset \mathcal{E}_{6} \subset \mathcal{K}_{6}, \quad \mathcal{A}_{5} \subset \mathcal{K}_{5} \subset \mathcal{K}_{5} \perp \mathcal{A}_{1} \subset \mathcal{K}_{6}, \\
& \mathcal{A}_{5} \subset \mathcal{A}_{6} \subset \mathcal{K}_{6}, \quad \mathcal{A}_{5} \subset \mathcal{D}_{6}^{(2)} \subset \mathcal{K}_{6}, \quad \mathcal{A}_{5} \subset \mathcal{D}_{6}^{(3)} \subset \mathcal{K}_{6}, \\
& \mathcal{A}_{6} \subset \mathcal{A}_{7} \subset \mathcal{E}_{7}, \quad \mathcal{A}_{7} \subset \mathcal{A}_{8} \subset \mathcal{E}_{8} \text { and } \quad \mathcal{E}_{7} \subset \mathcal{E}_{7} \perp \mathcal{A}_{1} \subset \mathcal{E}_{8} .
\end{aligned}
$$


Proof. We take $S$ to be a line system in $\mathbb{C}^{n+1}$, where $n=\operatorname{dim} T$. In all cases $\mathcal{A}_{2} \subset S$ and therefore, by Theorem 6.4, either $\mathcal{D}_{3}^{(2)} \subseteq S$ or $\mathcal{D}_{3}^{(3)} \subseteq S$.

If there is no subsystem of $S$ equivalent to $\mathcal{D}_{3}^{(3)}$, then by Theorem $8.4 S$ is Euclidean and the result follows from [5, Theorems 3.3.2 and 3.3.5].

Thus we may suppose that $\mathcal{D}_{3}^{(3)} \subseteq S$ and therefore, by Theorems 3.1 and 7.3, that $S$ is equivalent to $\mathcal{D}_{n}^{(3)}, \mathcal{D}_{n+1}^{(3)}, \mathcal{K}_{5}, \mathcal{K}_{6}$ or to a simple extension of $\mathcal{K}_{5}$ or $\mathcal{K}_{6}$.

If $S$ is equivalent to $\mathcal{D}_{m}^{(3)}$ for some $m$, then each element of $S$ is in a unique star with roots $a, b$ and $c$ such that $\Gamma_{a}=\emptyset$. It follows from Theorem 8.3 that stars ofthis type cannot wholly lie in $T$ and therefore $|T| \leq \frac{1}{3}\left|\mathcal{D}_{m}^{(3)}\right|=\left(\begin{array}{c}m \\ 2\end{array}\right)$. This eliminates all possibilities for $T$ except $\mathcal{A}_{n}$ and therefore the extension is $\mathcal{A}_{n} \subset \mathcal{D}_{n+1}^{(3)}$.

If $S$ is $\mathcal{K}_{5}$ or $\mathcal{K}_{6}$, then from Section 5 we see that all possible simple extensions exist, namely $\mathcal{A}_{4} \subset \mathcal{K}_{5}, \mathcal{A}_{5} \subset \mathcal{K}_{5}, \mathcal{A}_{5} \subset \mathcal{K}_{6}, \mathcal{A}_{6} \subset \mathcal{K}_{6}, \mathcal{K}_{5} \subset \mathcal{K}_{6}$ and $\mathcal{E}_{6} \subset \mathcal{K}_{6}$.

If $S$ is a simple extension of $\mathcal{K}_{5}$, then we may suppose, in the notation of Section 5, that $\overline{\mathcal{K}}_{5} \subset S \subset \mathbb{C}^{6}$. In particular, $S$ contains the 12 lines of $\mathcal{D}_{4}^{(2)}$, the line spanned by $u=(0,0,0,0, \sqrt{2}, 0)$ and the 32 images of the lines spanned by $v=\frac{1}{2}(1,1,1, i \sqrt{3}, \sqrt{2}, 0)$ under the action of $W\left(\mathcal{D}_{4}^{(2)}\right)$.

Suppose that $S$ is the star-closure of $\mathcal{K}_{5}$ and a root $x=\left(\alpha_{1}, \alpha_{2}, \ldots, \alpha_{6}\right)$. If $\alpha_{1}=\alpha_{2}=\alpha_{3}=\alpha_{4}=0$, then $(x, u)=\sqrt{2} \alpha_{5}$ and $(x, v)=(1 / \sqrt{2}) \alpha_{5}$, whence $\alpha_{5}=0$ and $S$ is equivalent to $\mathcal{K}_{5} \perp \mathcal{A}_{1}$. Therefore, if $\alpha_{i}=0$ for some $i \leq 4$, the argument of Lemma 7.1 shows that we may take $x$ to be $\left(1,0,0,0, \alpha_{5}, \alpha_{6}\right)$. Then $(x, u)=\sqrt{2} \alpha_{5}$ and $(x, v)=\frac{1}{2}+(1 / \sqrt{2}) \alpha_{5}$, whence $\alpha_{5}= \pm 1 / \sqrt{2}$. Thus, up to equivalence, $x=$ $(1,0,0,0,1 / \sqrt{2}, 1 / \sqrt{2})$. The unitary transformation $\mathbb{C}^{6} \rightarrow \mathbb{C}^{6}$ given by $e_{i} \mapsto e_{i}(1 \leq$ $i \leq 4), e_{5} \mapsto(1 / \sqrt{2})\left(e_{5}+e_{6}\right)$, and $e_{6} \mapsto(1 / \sqrt{2})\left(e_{5}-e_{6}\right)$ defines an equivalence between $S$ and $\overline{\mathcal{K}}_{6}$.

We may now suppose that $\alpha_{i} \neq 0$ for $i \leq 4$. A variation of the argument of Theorem 7.2 shows that we may assume that $\alpha_{1}=\alpha_{2}=\alpha_{3}$ $=\frac{1}{2}$ and that $\alpha_{4}$ is either $\frac{1}{2} \varepsilon$ or $\frac{1}{2} \varepsilon i \sqrt{3}$, where $\varepsilon= \pm 1$. If $x=\frac{1}{2}$ $\left(1,1,1, \varepsilon, 0,2 \alpha_{6}\right)$, then $(x, v)=\frac{1}{4}(3-\varepsilon i \sqrt{3})$, which is a contradiction. If $x=$ $\frac{1}{2}\left(1,1,1, \varepsilon i \sqrt{3}, 0,2 \alpha_{6}\right)$, then $(x, v)=\frac{3}{4}(1+\varepsilon)$ and so $\varepsilon=-1$ in this case. But $w=\frac{1}{2}(-1,1,1,-i \sqrt{3}, \sqrt{2}, 0)$ is a root of $S$ and $(x, w)=1$, hence $x-w=$ $\left(1,0,0,0,-1 / \sqrt{2}, \alpha_{6}\right)$ is also a root of $S$, reducing us to the previous case. Thus from now on we may suppose that $\alpha_{5} \neq 0$ and hence $\left|\alpha_{5}\right|=1 / \sqrt{2}$. If $\alpha_{4}=\varepsilon i \sqrt{3}$, then $\alpha_{6}=0$ and $(x, v)=\frac{1}{4}\left(3+3 \varepsilon+2 \sqrt{2} \alpha_{5}\right)$. It follows that $\varepsilon=-1$ and $\alpha_{5}= \pm 1 / \sqrt{2}$. But then $x \in \mathcal{K}_{5}$, which is a contradiction.

Thus we may suppose that $x=\frac{1}{2}\left(1,1,1, \varepsilon, 2 \alpha_{5}, 2 \alpha_{6}\right)$, whence $\left|\alpha_{5}\right|=\left|\alpha_{6}\right|$ $=1 / \sqrt{2}$ and so $(x, v)=\frac{1}{4}\left(3-\varepsilon i \sqrt{3}+2 \sqrt{2} \alpha_{5}\right)$. Consideration of the star of $x$ and $u$ shows that we are free to choose the sign of $\alpha_{5}$. Thus if $\varepsilon=1$ we may suppose that $\alpha_{5}=-\omega^{2} / \sqrt{2}$ and if $\varepsilon=-1$, we may take $\alpha_{5}=-\omega / \sqrt{2}$. Then $(x, v)=1$ in both cases. But then $x-v$ is a root whose first three coordinates are 0 and again we reduce 
to a previous case. Thus, up to equivalence, $\mathcal{K}_{6}$ is the only indecomposable simple extension of $\mathcal{K}_{5}$.

Finally, suppose that $S$ is the star-closure of $\mathcal{K}_{6}$ and a root $x=\left(\alpha_{1}, \alpha_{2}, \ldots, \alpha_{7}\right)$ in $\mathbb{C}^{7}$. We have $\mathcal{D}_{6}^{(3)} \subset \mathcal{K}_{6}$ and the star-closure of $\mathcal{D}_{6}^{(3)}$ and $x$ is indecomposable. From Theorem 7.3 the only indecomposable simple extensions of $\mathcal{D}_{6}^{(3)}$ are $\mathcal{D}_{7}^{(3)}$ and $\mathcal{K}_{6}$. Furthermore, if $\mathcal{D}_{7}^{(3)} \subseteq S$, then equality holds, since there are no indecomposable extensions of $\mathcal{D}_{7}^{(3)}$ in $\mathbb{C}^{7}$ (Theorem 7.3). But then $\left|\mathcal{K}_{6}\right| \leq\left|\mathcal{D}_{7}^{(3)}\right|$, which is a contradiction.

From the proof of case $n=6$ of Theorem 7.3 we see that $\alpha_{7}=0$, hence $S$ is contained in $\mathbb{C}^{6}$. In $\mathbb{C}^{6}$ there are just three extensions of $\mathcal{D}_{6}^{(3)}$ equivalent to $\mathcal{K}_{6}$ : the star-closures of $\mathcal{D}_{6}^{(3)}$ with $\theta^{-1}(1,1,1,1,1,1), \theta^{-1}(1,1,1,1,1, \omega)$ and $\theta^{-1}\left(1,1,1,1,1, \omega^{2}\right)$, respectively, where $\theta=\omega-\omega^{2}$. On the other hand, no two of the roots just given are at $60^{\circ}$ or $90^{\circ}$. Therefore, $\mathcal{K}_{6}$ has no indecomposable simple extensions.

The list of simple extensions that are not minimal follows from the descriptions of the line systems given in Sections 4 and 5.

An immediate consequence of the classification of extensions of line systems is the following theorem, essentially due to Mitchell [9], for line systems of primitive reflection groups.

THEOREM 9.2. If $S$ is an indecomposable star-closed line system, then $S$ is equivalent to $\mathcal{A}_{n}, \mathcal{D}_{n}^{(2)}, \mathcal{D}_{n}^{(3)}, \mathcal{E}_{6}, \mathcal{E}_{7}, \mathcal{E}_{8}, \mathcal{K}_{5}$ or $\mathcal{K}_{6}$

\section{References}

[1] N. Bourbaki, Groupes et algèbres de Lie, Chapitres 4, 5 et 6 (Hermann, Paris, 1968).

[2] P. J. Cameron, J.-M. Goethals, J. J. Seidel and E. E. Shult, 'Line graphs, root systems, and elliptic geometry', J. Algebra 43(1) (1976), 305-327.

[3] Arjeh M. Cohen, 'Finite complex reflection groups', Ann. Sci. Ecole Norm. Sup. (4) 9(3) (1976), $379-436$.

[4] J. H. Conway, R. T. Curtis, S. P. Norton, R. A. Parker and R. A. Wilson, Atlas of Finite Groups (Oxford University Press, Eynsham, 1985).

[5] Dragoš Cvetković, Peter Rowlinson and Slobodan Simić, Spectral Generalizations of Line Graphs, London Mathematical Society Lecture Note Series, 314 (Cambridge University Press, Cambridge, 2004).

[6] P. Delsarte, J. M. Goethals and J. J. Seidel, 'Bounds for systems of lines, and Jacobi polynomials', Philips Res. Rep. 30 (1975), 91-105.

[7] William M. Kantor, 'Generation of linear groups', in: The Geometric Vein: The Coxeter Festschrift (eds. Chandler Davis, Branko Grünbaum and F. A. Sherk) (Springer, New York, 1981), pp. 497-509.

[8] Tom H. Koornwinder, 'A note on the absolute bound for systems of lines', Indag. Math (N.S.) 38 (1976), 152-153.

[9] H. H. Mitchell, 'Determination of all primitive collineation groups in more than four variables which contain homologies', Amer. J, Math. 36 (1914), 1-12.

[10] G. C. Shephard and J. A. Todd, 'Finite unitary reflection groups', Canad. J. Math. 6 (1954), 274-304. 
MURALEEDARAN KRISHNASAMY, School of Mathematics and Statistics, University of Sydney, Australia

and

Current address: United Arab Emirates University, P.O. Box 15551, Al-Ain, United Arab Emirates

e-mail:muraleek@uaeu.ae

D. E. TAYLOR, School of Mathematics and Statistics, University of Sydney, Australia e-mail: D.Taylor@maths.usyd.edu.au 